\title{
Comparison of Two Different Treatment Methods to Treat the Temporomandibular Joint Disorders (Myofascial Pain) in a Short Period of Time - A Retrospective Study
}

\author{
Hamad Nasser Albageah ${ }^{1}$, Abdulaziz Abdulhakim Alwakeel ${ }^{2}$ \\ 1, 2 Department of Oral and Diagnostic Science, Dental Collage, King Saud University, Riyadh, Saudi Arabia.
}

\section{ABSTRACT}

\section{BACKGROUND}

Temporomandibular joint (TMJ) is the third most common site of pain in the orofacial area, while the masseter muscle was the primary painful masticatory muscle. The temporal and frontal region were pain areas indicated by temporomandibular joint disorder (TMDs) patients. The purpose of this study was to compare two different treatment modalities, physical therapy and occlusal appliance to treat myofascial pain.

\section{METHODS}

This retrospective study comprises of all orofacial pain patients attending orofacial pain clinics of Dental University Hospital, King Saud University in Riyadh, Saudi Arabia. Patients were categorized into two groups, the first group: patients treated by the occlusal appliance (hard type). The second group: patients treated by physiotherapy home exercises, including posture position modification. Physical therapy included muscle stretching and isometric tension against resistance exercises and guided jaw movements. Methods of clinical examination was based on the research diagnostic criteria for temporomandibular disorders (RDC/TMD) criteria. The data of pain level was collected based on the visual analog scale (VAS).

\section{RESULTS}

$16.1 \%$ of patients were male, and $83.9 \%$ of the patients were female. With the mean age being 31.1 years old. $92.9 \%$ were Saudi patients and $7.1 \%$ were non-Saudi. 50 $\%$ of the patients were using an occlusal appliance, and $50 \%$ went for physiotherapy. The independent t-test showed a highly significant difference between different management methods with a P - value of 0.038 and a mean difference of 0.32143 . $80.5 \%$ of the patients reported masseter muscle pain as one of their main complaints.

\section{CONCLUSIONS}

A significant difference was observed between physiotherapy and occlusal appliances with education in treating patients with myofascial pain. Patients using the occlusal appliances showed a high percentage of pain reduction (85.7\%) compared to physiotherapy treatment $(57.1 \%)$ in a short period of time. Henceforth, patient's education plays a significant role in pain reduction.

\section{KEY WORDS}

Temporomandibular Joint Disorder, Occlusal Appliance, Myofascial Pain, Physiotherapy
Corresponding Author: Dr. Hamad Nasser Albageah, Dental Collage, King Saud University, Riyadh, Saudi Arabia.

E-mail: halbageah@ksu.edu.sa

DOI: $10.14260 / \mathrm{jemds} / 2021 / 678$

How to Cite This Article:

Albageah HN, Alwakeel AA. Comparison of two different treatment methods to treat the temporomandibular joint disorders (myofascial pain) in a short period of time a retrospective study. J Evolution Med Dent Sci 2021;10(38):3342-3345, DOI: $10.14260 / j e m d s / 2021 / 678$

Submission 26-05-2021,

Peer Review 30-08-2021,

Acceptance 06-09-2021, Published 20-09-2021.

Copyright (C) 2021 Hamad Nasser Albageah et al. This is an open access article distributed under Creative Commons Attribution License [Attribution 4.0 International (CC BY 4.0)] 


\section{BACKGROUND}

Temporomandibular disorder is a word that refers to a large number of clinical abnormalities that involve multiple structure in TMJ area such as muscle, joint (TMJ), or both and the associated structures. ${ }^{1}$

TMJ is considered as the third most common site of pain, while the masseter muscle being the painful site of all the masticatory muscle. ${ }^{2}$ The aetiology of TMJ pain is multifactorial. It could be related to mallocclusal, bone, muscle structure and function abnormality. ${ }^{3}$ Anatomic correlations between the craniomandibular complex and the cervical spine have been assessed in some studies. ${ }^{4}$ Non-invasive treatments are used to decrease muscle spasms during jaw movements. Several studies have suggested that physiotherapy treatment is helpful to treat TMDs. ${ }^{5}$ Physical therapy plays a role in managing masticatory muscles and joints pain, ${ }^{6}$ including selfcare, improve patient awareness and modified the life habits. ${ }^{7}$ Physical therapy for TMDs includes TMJ and soft tissue mobilization of painful muscles, stretching of the masticatory muscles, exercises, isometric tension against resistance exercises. ${ }^{8}$ The occlusal appliances (OAs) approach has evolved with different designs and claims of efficacy. A recent study review stated that OAs might be an effective management modality to treat the TMDs as a placebo treatment rather than a specific therapeutic mechanism. ${ }^{9}$ This study aimed to compare two different treatment modalities, physical therapy and occlusal appliance, to treat myofascial pain.

\section{METHODS}

This retrospective study comprises all orofacial pain patients attending orofacial pain clinics of Dental University Hospital, King Saud University in Riyadh, Saudi Arabia from October 2020 to February 2021. Fifty-six patients were categorized into two groups, the first group: twenty-eight patients treated by the occlusal appliance (hard type). ${ }^{10}$ The second group: twenty-eight patients treated by physiotherapy home exercises, including posture position modification. Physical therapy included muscle stretching and isometric tension against resistance exercises and guided jaw movements. ${ }^{8}$ Methods of clinical examination based on RDC/TMD criteria. ${ }^{11}$ Both groups were educated to stop any parafunctional habit such as chewing hard food and opening mouth widely.

This study was approved by the institutional review board in medical city of King Saud University, Riyadh, Saudi Arabia project number E-20-5405.

The data was collected for all patients with tenderness in the muscles of mastication bilateral and shoulder and neck area. ${ }^{12,13}$

The level of pain during clinical examinations was collected based on the visual analog scale from the first visit and after two weeks of using different treatment modalities. ${ }^{14}$ The exclusion criteria included:

- Patients with known history of surgery.

- Any major head or neck trauma, current orthodontic treatment.

- A diagnosis of rheumatoid arthritis or autoimmune diseases.

\section{Statistical Analysis}

The statistical analysis was done by the Statistical Package for Social Sciences (SPSS v20) and the independent t-test was used at the level of significance $\mathrm{P}<0.05$. The power of the sample of this study was more than 80 .

\section{RESULTS}

The results showed $16.1 \%$ of the patients were males and 83.9 $\%$ were females. The age ranged from 13 to 68 years old with mean 31.1 years and standard deviation 13.26. $92.9 \%$ were Saudi patients, and $7.1 \%$ were non-Saudi. $50 \%$ of the patient has used occlusal appliances, and $50 \%$ went for physiotherapy.

During the clinical examination before the treatment, $69.64 \%$ of patients reported pain in all muscles of mastication, where $21.14 \%$ was in the masseter muscle and $9 \%$ in temporalis muscle only. All patients showed no bone pathology or changes in the condyle of the mandible based on radiographic evaluation.

\begin{tabular}{|cccc|}
\hline Level of Pain/ Treatment Method & Increased & Same & Decreased \\
Occlusal appliances (OAs) & $3.6 \%(1)$ & $10.7 \%(3)$ & $85.7 \%(24)$ \\
Physiotherapy (PT) & $7.1 \%(2)$ & $35.7 \%(10)$ & $57.1 \%(16)$ \\
\hline Table 1. Number and Percentage of Patients Who Report the Level \\
of Pain on the VAS Scale after Two Weeks of Treatment \\
\hline
\end{tabular}

\begin{tabular}{|cccc|}
\hline $\begin{array}{c}\text { The Mean Score of } \\
\text { (VAS) Out of } \mathbf{1 0} \text { and } \\
\text { Standard } \\
\begin{array}{c}\text { Deviation/Type of } \\
\text { Muscle }\end{array}\end{array}$ & $\begin{array}{c}\text { Before } \\
\text { Treatment for } \\
\text { All of Muscles } \\
\text { of Mastication }\end{array}$ & $\begin{array}{c}\text { After Treating } \\
\text { Patients by } \\
\text { Occlusal } \\
\text { Appliance }\end{array}$ & $\begin{array}{c}\text { Patients by } \\
\text { Physiotherapy }\end{array}$ \\
Masseter & $6.01(1.37)$ & $3.51(1.67)$ & $4.75(1.76)$ \\
Temporalis & $4.21(2.40)$ & $1.35(1.52)$ & $3.39(2.12)$ \\
\hline Table 2. The Mean Score of (VAS) and Standard Deviation before \\
and after Treatment of All Patients During Palpation of Muscles of \\
Mastication (Masseter and Temporalis)
\end{tabular}

The independent $t$-test showed a statistically significant difference in level of pain between patients who used occlusal appliance and physiotherapy with $\mathrm{P}$ value of 0.038 , a mean difference of 0.32143 and a $95 \%$ confidence interval of the difference [0.01927: 0.062358]

\section{DISCUSSION}

Masticatory myalgia has been divided into three subtypes:

1. Local myalgia, which is characterized as local pain in the site of muscle palpation.

2. Myofascial pain, which is characterized as distributed pain around the area of palpated muscle.

3. Myofascial pain with referral is described as pain that refers from one site to another at a place beyond the muscle being palpated.

In a diagnosis of myalgia, the patient continuously mentioned pain in the orofacial area, and the pain level increased during jaw movement. In addition, the pain is usually localized in the temporalis or masseter muscles. ${ }^{11}$ If the signs and symptoms presented for three months, it indicated that the patient has chronic pain. ${ }^{15}$

According to the RDC $\backslash \mathrm{TMD}$, the diagnosis of myalgia includes pain during mouth opening and the palpation of the 
temporalis and masseter regions. So, in this study, $80.5 \%$ of the patients reported pain, especially in the masseter muscle. In contrast, the other masticatory muscles have shown a low percentage of pain which highly indicates the diagnosis of myalgia 11

The level of pressure during the palpation to provoke this pain is $1 \mathrm{~kg} \backslash 2 \mathrm{sec}$. However, to differentiate the types of myalgia, palpation pressure of $1 \mathrm{~kg}$ needs to be continued at least for 5 seconds at the area of muscle to produce referred pain. ${ }^{11}$

Myofascial pain in the orofacial area is the most common diagnosis in patients with orofacial pain (45.3\%). ${ }^{16}$ Myofascial pain syndrome (MPS) is defined as neurological symptoms caused by myofascial TrPs. The best aetiology presented until now that found the TrPs have been developed after muscle function overloaded.11

TrPs referred pain type, and a local twitch muscle response can be prompted by mechanical stimulation (palpation or needle insertion). The trigger point is usually activated by injury of muscle and surrounded structure. ${ }^{17}$

The treatment of MPS is usually by medications followed by physiotherapy and occlusal splint therapy. Laser therapy can also be used. The analgesics and anti-inflammatory medication were the most common medication used to treat the MPS. 18

Physiotherapy treatment can be used by applying deepstroking massage to the TrPs. ${ }^{19}$ The psychological situation of the patient who has MPS should be managed because the psychosocial factors can be driving MPS.20 Some clinical studies suggest that OAs show more effective results for treating myogenic conditions more than they are for intracapsular disorders. But it can be helpful to manage both conditions. ${ }^{9}$

A study carried out by Michelotti et al. showed that after three months, changes in spontaneous muscle pain significantly decreased in patients who had education more than the patients who had occlusal splints with statistically significant differences between the two groups. $(\mathrm{P}=.034$; effect size $=0.33$ ).

This study result shows highly significant differences in pain reduction of the group that used occlusal splint with education, thus emphasizing that the combination of occlusal splint and education can significantly reduce the pain. ${ }^{21}$

Another study demonstrated the effectiveness of the prefabricated occlusal appliances to be same as that of the stabilization appliances. The prefabricated appliance can be used as a short-term therapy in young patients with myofascial pain. ${ }^{10}$

Furthermore, Amin et al. compared different occlusal appliances, hard splint, soft splint, and liquid oral splint, in all three groups. A statistically significant reduction of pain was found in all groups of study at the end of 3 months.

However, the hard type of occlusal appliance showed a very effective result in a shorter period, followed by liquid and soft occlusal appliance. In the present study, the rigid type of occlusal appliance was used.

According to the VAS scale, high significant pain reduction was detected, similar to previous studies where the pain was found to reduce in a short period. ${ }^{22}$ Similarly, in a previous study, Michelotti et al. showed the effectiveness of physiotherapy in myofascial pain over three months, where significant differences $(\mathrm{P}=.019)$ were noted between physiotherapy with education group and education group only. Within the present findings, patients who followed physiotherapy and education had muscle pain reduction (57.1 $\%)$ lower than the group that used occlusal appliances and education $(85.7 \%)$.

The short period of patient evaluation in this study may not represent the actual effect of physiotherapy as presented in Michelotti et al. study. ${ }^{23}$

\section{CONCLUSIONS}

A significant difference was observed between physiotherapy and occlusal appliances with education in treating patients with myofascial pain. Patients using the occlusal appliances showed a high percentage of pain reduction (85.7\%) compared to physiotherapy treatment $(57.1 \%)$ in a short period of time. Henceforth, patient's education plays a significant role in pain reduction.

\section{Limitation of Study}

This study showed several limitations. The first limitation was focusing on myofascial pain only. The second limitation was that some studies showed a significant effect of physiotherapy in long term follow-up patients. Still, in this study, the followup appointment of patients was during a short period compared to previous studies.

Data sharing statement provided by the authors is available with the full text of this article at jemds.com.

Financial or other competing interests: None.

Disclosure forms provided by the authors are available with the full text of this article at jemds.com.

\section{REFERENCES}

[1] Clark GT, Green EM, Dornan MR, et al. Craniocervical dysfunction levels in a patient sample from a temporomandibular joint clinic. J Am Dent Assoc 1987;115(2):251-6.

[2] Pedroni CR, de Oliveira AS, Bérzin F. Pain characteristics of temporomandibular disorder: a pilot study in patients with cervical spine dysfunction. J Appl Oral Sci 2006;14(5):388-92.

[3] Parker MW. A dynamic model of etiology in temporomandibular disorders. J Am Dent Assoc 1990;120(3):283-90.

[4] Solow B, Sandham A. Crânio-cervical posture: a factor in the development and function of the dentofacial structures. Eur J Orthod 2002;24(5):447-56.

[5] Feine JS, Lund JP. An assessment of the ef cacy of physical therapy and physical modalities for the control of chronic musculoskeletal pain. Pain 1997;71(1):5-23.

[6] Michelotti A, Stinks MH, Ferrule M, et al. The additional value of a home physical therapy regimen versus patient education only for the treatment of myofascial pain of the jaw muscles: short-term results of a randomized clinical trial. J Orofac Pain 2004;18(2):114-25. 
[7] Okeson JP. Management of temporomandibular disorders and occlusion. $5^{\text {th }}$ edn. St. Louis: Mosby 2003: p. 371-93.

[8] Hanten WP, Olson SL, Butts NL, et al. Effectiveness of a home program of ischemic pressure followed by sustained stretch for treatment of myofascial trigger points. Phys Ther 2000;80(10):997-1003.

[9] Klasser GD, Greene CS. Oral appliances in the management of temporomandibular disorders. Oral Surg Oral Med Oral Pathol Oral Radiol Endodontol 2009;107(2):212-23.

[10] Nilner M, Ekberg E, Doepel M, et al. Short-term effectiveness of a prefabricated occlusal appliance in patients with myofascial pain. J Orofac Pain 2008;22(3):209-18.

[11] Schiffman E, Ohrbach R, Truelove E, et al. Diagnostic criteria for temporomandibular disorders (DC/TMD) for Clinical and Research Applications: recommendations of the International RDC/TMD Consortium Network and Orofacial Pain Special Interest Group. J Oral Facial Pain Headache 2014;28(1):6-27.

[12] Mongini F, Ciccone G, Ceccarelli M, et al. Muscle tenderness in di erent types of facial pain and its relation to anxiety and depression: a cross-sectional study on 649 patients. Pain 2007;131(1-2):106-11.

[13] AlghAdir A, Zafar H, Iqbal ZA. Work-related musculoskeletal disorders among dental professionals in Saudi Arabia. J Phys Ther Sci 2015;27(4):1107-12.

[14] Munro BH. Statistical methods for health care research. $5^{\text {th }}$ edn. Philadelphia, Pa, USA: Lippincott Williams \& Wilkins 2005.
[15] Maixner W, Fillingim R, Sigurdsson A, et al. Sensitivity of patients with painful temporomandibular disorders to experimentally evoked pain: evidence for altered temporal summation of pain. Pain 1998;76(1-2):71-81.

[16] Manfredini D, Guarda-Nardini L, Winocur E, et al. Research diagnostic criteria for temporomandibular disorders: a systematic review of axis I epidemiologic findings. Oral Surg Oral Med Oral Pathol Oral Radiol Endodontol 2011;112(4):453-62.

[17] Hong CZ. Pathophysiology of myofascial trigger point. J Formos Med Assoc 1996;95(2):93-104.

[18] Pal US, Kumar L, Mehta G, et al. Trends in management of myofacial pain. Natl J Maxillofac Surg 2014;5(2):109-16.

[19] Davies C. The trigger point therapy workbook. $2^{\text {nd }}$ edn. Oakland: New Harbinger Publication Inc., 2004: p. 47-74.

[20] Lee M, Kim M, Oh S, et al. A self-determination theorybased self-myofascial release program in older adults with myofascial trigger points in the neck and back: a pilot study. Physiother Theory Pract 2017;33(9):681-94.

[21] Michelotti A, Iodice G, Vollaro S, et al. Evaluation of the short-term effectiveness of education versus an occlusal splint for the treatment of myofascial pain of the jaw muscles. J Am Dent Assoc 2012;143(1):47-53.

[22] Amin A, Meshramkar R, Lekha K. Comparative evaluation of clinical performance of different kind of occlusal splint in management of myofascial pain. J Indian Prosthodont Soc 2016;16(2):176-81. 